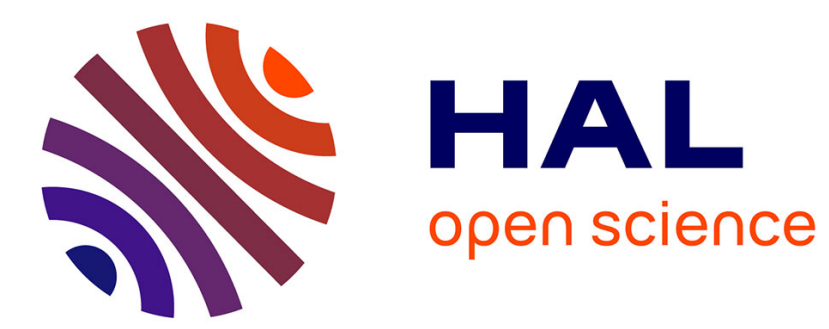

\title{
The prime number theorem in short intervals for automorphic L-functions \\ $\mathrm{Y} \mathrm{Qu}, \mathrm{J} \mathrm{Wu}$
}

\section{To cite this version:}

$\mathrm{Y} \mathrm{Qu}, \mathrm{J} \mathrm{Wu}$. The prime number theorem in short intervals for automorphic L-functions. Acta Arithmetica, 2012, 10.4064/aa154-1-3 . hal-01278404

\section{HAL Id: hal-01278404 \\ https://hal.science/hal-01278404}

Submitted on 24 Feb 2016

HAL is a multi-disciplinary open access archive for the deposit and dissemination of scientific research documents, whether they are published or not. The documents may come from teaching and research institutions in France or abroad, or from public or private research centers.
L'archive ouverte pluridisciplinaire HAL, est destinée au dépôt et à la diffusion de documents scientifiques de niveau recherche, publiés ou non, émanant des établissements d'enseignement et de recherche français ou étrangers, des laboratoires publics ou privés. 


\title{
THE PRIME NUMBER THEOREM IN SHORT INTERVALS FOR AUTOMORPHIC L-FUNCTIONS
}

\author{
Y. QU \& J. WU
}

\begin{abstract}
In this paper, we generalize Selberg's normal density theorem in short intervals [12] and Heath-Brown's refinement [2] to the case of automorphic $L$ functions of $G L_{m}\left(\mathbb{A}_{\mathbb{Q}}\right)$.
\end{abstract}

\section{INTRODUCTION}

The well known Legendre conjecture states that there is at least one prime number between $n^{2}$ and $(n+1)^{2}$ for each positive integer $n$. A related problem is the existence of primes in short intervals. Denote, as usual, by $\zeta(s)$ the Riemann zeta-function, and define the von Mangoldt function $\Lambda(n)$ by

$$
-\frac{\zeta^{\prime}}{\zeta}(s)=\sum_{n=1}^{\infty} \frac{\Lambda(n)}{n^{s}} \quad(\sigma>1),
$$

where $s=\sigma+\mathrm{i} \tau$. Then

$$
\Lambda(n)= \begin{cases}\log p & \text { if } n=p^{\nu} \text { with } \nu \geqslant 1 \\ 0 & \text { otherwise. }\end{cases}
$$

Write

$$
\psi(x):=\sum_{n \leqslant x} \Lambda(n)
$$

It is known that, under the Riemann Hypothesis ( $\mathrm{RH}$ in brief) for $\zeta(s)$,

$$
\psi(x)=x+O\left(x^{1 / 2}(\log x)^{2}\right) \quad(x \geqslant 2) .
$$

From this we immediately deduce that, under $\mathrm{RH}$,

$$
\psi(x+h(x))-\psi(x) \sim h(x) \quad(x \rightarrow \infty)
$$

for any increasing functions $h(x) \leqslant x$ satisfying

$$
\frac{h(x)}{x^{1 / 2}(\log x)^{2}} \rightarrow \infty \quad \text { as } \quad x \rightarrow \infty .
$$

It seems be an interesting problem to determine how short $h(x)$ can be. According to Cramér's model, we could take $h(x) /(\log x)^{2} \rightarrow \infty$ as $x \rightarrow \infty$. In 1943, Selberg

Date: April 13, 2012.

2000 Mathematics Subject Classification. 11F30, 11F11, 11F66.

Key words and phrases. Automorphic $L$-function, normal density theorem, short interval, Riemann Hypothesis. 
[12] partially confirmed this by showing that under RH asymptotic relationship

$$
\int_{1}^{X}|\psi(x+h(x))-\psi(x)-h(x)|^{2} \mathrm{~d} x=o\left(h(X)^{2} X\right) \quad(X \rightarrow \infty)
$$

holds for any increasing functions $h(x) \leqslant x$ satisfying

$$
\frac{h(x)}{(\log x)^{2}} \rightarrow \infty \quad \text { as } \quad x \rightarrow \infty
$$

It shows that, under RH, (1.1) holds for almost all $x \geqslant 2$ provided (1.3) is satisfied.

In order to better understand the connection between the distribution of zeros of $\zeta(s)$ and that of primes, Montgomery [9] introduced the pair correlation function

$$
F_{T}(X):=\sum_{0<\gamma_{1}, \gamma_{2} \leqslant T} W\left(\gamma_{1}-\gamma_{2}\right) \mathrm{e}\left(X\left(\gamma_{1}-\gamma_{2}\right)\right)
$$

where

$$
W(u):=\frac{4}{4+u^{2}}, \quad \mathrm{e}(u):=\mathrm{e}^{2 \pi \mathrm{i} u},
$$

and $\gamma$ runs over imaginary parts $\Im m \rho$ of the nontrivial zeros $\rho$ of $\zeta(s)$ (counted according to multiplicity). Assuming RH and that

$$
F_{T}\left(\frac{\log x}{2 \pi}\right) \ll T \log T
$$

uniformly for $x(\log x)^{-3} \leqslant T \leqslant x$, Heath-Brown [2] showed that (1.2) holds for any increasing functions $h(x) \leqslant x$ satisfying

$$
\frac{h(x)}{\log x} \rightarrow \infty \quad \text { as } \quad x \rightarrow \infty
$$

In this paper, we shall investigate analogues of (1.2) for automorphic $L$-functions. Let us fix our notation first. To each irreducible unitary cuspidal representation $\pi=\otimes \pi_{p}$ of $G L_{m}\left(\mathbb{A}_{\mathbb{Q}}\right)$ with $m \geqslant 2$, one can attach a global $L$-function

$$
L(s, \pi)=\prod_{p<\infty} L_{p}\left(s, \pi_{p}\right)
$$

converging for $\sigma>1$ (see [5]), where local factors are given by

$$
L_{p}\left(s, \pi_{p}\right)=\prod_{j=1}^{m}\left(1-\alpha_{\pi}(p, j) p^{-s}\right)^{-1} .
$$

The complete $L$-function $\Phi(s, \pi)$ is defined by

$$
\Phi(s, \pi)=L(s, \pi) L_{\infty}\left(s, \pi_{\infty}\right),
$$

where $N_{\pi} \geqslant 1$ is an integer called the arithmetic conductor of $\pi$, and

$$
L_{\infty}\left(s, \pi_{\infty}\right):=\left(\frac{N_{\pi}}{\pi^{m}}\right)^{s / 2} \prod_{j=1}^{m} \Gamma\left(\frac{s+\mu_{\pi}(j)}{2}\right)
$$

is the Archimedean local factor. Here $\left\{\alpha_{\pi}(p, j)\right\}_{j=1}^{m}$ and $\left\{\mu_{\pi}(j)\right\}_{j=1}^{m}$ are complex numbers associated with $\pi_{p}$ and $\pi_{\infty}$, respectively, according to the Langlands correspondence. Good bounds for these local parameters are of fundamental importance 
for the study of automorphic $L$-functions. Thanks to the work of Luo-RudnickSarnak [8], it is known that

$$
\begin{cases}\left|\alpha_{\pi}(p, j)\right| \leqslant p^{\theta} & \text { if } \pi \text { is unramified at } p \\ \left|\Re e \mu_{\pi}(j)\right| \leqslant \theta & \text { if } \pi \text { is unramified at } \infty\end{cases}
$$

with $\theta=1 / 2-1 /\left(m^{2}+1\right)$. The Generalized Ramanujan Conjecture (GRC in brief) asserts that (1.11) should hold with $\theta=0$. It also follows from work of Shahidi $[13,14,15,16]$ that the complete $L$-function $\Phi(s, \pi)$ has an analytic continuation to the whole complex plane and satisfies the functional equation

$$
\Phi(s, \pi)=\varepsilon_{\pi} \Phi(1-s, \tilde{\pi}),
$$

where $\varepsilon_{\pi}$ is the root number satisfying $\left|\varepsilon_{\pi}\right|=1$, and $\tilde{\pi}$ is the representation contragredient to $\pi$. Iwaniec and Sarnak [4] introduced the analytic conductor of $\pi$. It is a function over the reals given by

$$
Q_{\pi}(t)=N_{\pi} \prod_{j=1}^{m}\left(3+\left|t+\mu_{\pi}(j)\right|\right),
$$

which puts together all the important parameters for $\pi$. The quantity

$$
Q_{\pi}=Q_{\pi}(0)=N_{\pi} \prod_{j=1}^{m}\left(3+\left|\mu_{\pi}(j)\right|\right)
$$

is also important, and it is named the conductor of $\pi$.

Similarly to the classical case, we define $\Lambda_{\pi}(n)$ by taking logarithmic differentiation in (1.7)

$$
-\frac{L^{\prime}}{L}(s, \pi)=\sum_{n=1}^{\infty} \frac{\Lambda_{\pi}(n)}{n^{s}} \quad(\sigma>1) .
$$

With the help of (1.8), it is easy to see that

$$
\Lambda_{\pi}(n)= \begin{cases}\sum_{j=1}^{m} \alpha_{\pi}(p, j)^{\nu} \log p & \text { if } n=p^{\nu} \text { with } \nu \geqslant 1 \\ 0 & \text { otherwise. }\end{cases}
$$

The prime number theorem for $L(s, \pi)$ concerns the asymptotic behavior of the counting function

$$
\psi(x, \pi):=\sum_{n \leqslant x} \Lambda_{\pi}(n)
$$

This problem was first studied by Liu \& Ye [7] and $\mathrm{Qu}[10,11]$. In particular $\mathrm{Qu}[11]$ proved that, under the Generalized Riemann Hypothesis (GRH in brief) for $L(s, \pi)$, we have

$$
\int_{1}^{X}|\psi(x+h(x), \pi)-\psi(x, \pi)|^{2} \mathrm{~d} x=o\left(h(X)^{2} X\right) \quad(X \rightarrow \infty)
$$

for any increasing functions $h(x) \leqslant x$ satisfying

$$
\frac{h(x)}{x^{\theta}(\log x)^{2}} \rightarrow \infty \quad \text { as } \quad x \rightarrow \infty
$$


where $\theta$ is given by (1.11).

The first aim of this paper is to improve the above result by removing $x^{\theta}$, which offers an exact generalization of Selberg's (1.2) and (1.3) to automorphic $L$-functions.

thm 1. Let $\pi$ be an irreducible unitary cuspidal representation of $G L_{m}\left(\mathbb{A}_{\mathbb{Q}}\right)$ with $m \geqslant 2$. Assume $G R H$ for $L(s, \pi)$. Then for $X \geqslant 2$ we have

$$
\int_{1}^{X}|\psi(x+h(x), \pi)-\psi(x, \pi)|^{2} \mathrm{~d} x \ll h(X) X \log ^{2}\left(Q_{\pi} X\right)+\left(\frac{\log Q_{\pi}}{\log X}\right)^{4}
$$

for any increasing functions $h(x) \leqslant x$, where the implied constant depends only on $m$. In particular, (1.16) holds for any increasing functions $h(x) \leqslant x$ satisfying

$$
\frac{h(x)}{(\log x)^{2}} \rightarrow \infty \quad \text { as } \quad x \rightarrow \infty
$$

Our second aim of this paper is to consider the analogue of Heath-Brown's (1.2) and (1.6). Similar to (1.4), we can also define

$$
F_{T}^{\pi}(X):=\sum_{0<\gamma_{1}, \gamma_{2} \leqslant T} W\left(\gamma_{1}-\gamma_{2}\right) \mathrm{e}\left(X\left(\gamma_{1}-\gamma_{2}\right)\right)
$$

where $\gamma$ runs over imaginary parts $\Im m \rho$ of the nontrivial zeros $\rho$ of $L(s, \pi)$ (counted according to multiplicity).

thm 2. Let $\pi$ be an irreducible unitary cuspidal representation of $G L_{m}\left(\mathbb{A}_{\mathbb{Q}}\right)$ with $m \geqslant 2$. Assume $G R H$ for $L(s, \pi)$, and

$$
F_{T}^{\pi}\left(\frac{\log X}{2 \pi}\right) \ll T \log \left(Q_{\pi} T\right)
$$

uniformly for $T \leqslant(X \log X)^{2}$. Then for $X \geqslant 2$ we have

$$
\begin{aligned}
\int_{1}^{X}|\psi(x+h(x), \pi)-\psi(x, \pi)|^{2} \mathrm{~d} x \ll & h(X) X \log \left(Q_{\pi} X\right) \\
& +X \log ^{2}\left(Q_{\pi} X\right)+\left(\frac{\log Q_{\pi}}{\log X}\right)^{4}
\end{aligned}
$$

for any increasing functions $h(x) \leqslant x$, where the implied constant depends only on $m$. In particular, (1.16) holds for any increasing functions $h(x) \leqslant x$ satisfying

$$
\frac{h(x)}{\log x} \rightarrow \infty \quad \text { as } \quad x \rightarrow \infty
$$

Theorem 1 (resp. Theorem 2) shows that, under GRH (resp. under GRH and (1.19)) for almost all $x \geqslant 2$, we have

$$
\psi(x+h(x), \pi)-\psi(x, \pi)=o(h(x)) \quad(x \rightarrow \infty),
$$

provided (1.18) (resp. (1.21)) is satisfied. It means that the sequence $\left\{\Lambda_{\pi}(n)\right\}_{n \geqslant 1}$ changes signs (different from the classical case $\{\Lambda(n)\}_{n \geqslant 1}$ ). Very recently, Liu, Qu $\& \mathrm{Wu}$ [6] showed that if $\Lambda_{\pi}(n)$ is real for all $n \geqslant 1$, then there is some $n$ satisfying

$$
n \ll_{m, \varepsilon} Q_{\pi}^{1+\varepsilon}
$$


such that $\Lambda_{\pi}(n)<0$. The implied constant depends only on $m$ and $\varepsilon$. In particular, this result is true for any self-contragredient irreducible unitary cuspidal representation $\pi$ for $G L_{m}\left(\mathbb{A}_{\mathbb{Q}}\right)$ with trivial central character.

The main new ideas for proving Theorems 1 and 2 are a delicate application of Iwaniec-Kowalski's mean value estimate (cf. (3.3) below) and an explicit formula in a more precise form adapted to our purpose (cf. Lemma 3.1 below).

Acknowledgements. This work has been finished during the second author's visit to the Morningside Center of Mathematics of Chinese Academy of Sciences, whose financial support and hospitality are gratefully acknowledged.

\section{Preliminary Lemmas}

In view of (1.10) and the fact that $L(s, \pi)$ and $\Phi(s, \pi)$ are entire, it is not difficult to see that the trivial zeros of $L(s, \pi)$ and the poles of $L_{\infty}\left(1-s, \tilde{\pi}_{\infty}\right)$ are

$$
\begin{aligned}
\mu & :=-2 n-\mu_{\pi}(j) \text { for } n=0,1,2, \ldots ; j=1, \ldots, m, \\
P_{n, j} & :=2 n+1+\mu_{\widetilde{\pi}}(j) \text { for } n=0,1,2, \ldots ; j=1, \ldots, m,
\end{aligned}
$$

respectively. As in [7], we let $\mathbb{C}(m)$ denote the complex plane with the discs

$$
\left|s-P_{n, j}\right|<(8 m)^{-1} \text { for } n=0,1,2, \ldots ; j=1, \ldots, m
$$

excluded. Thus, for any $s \in \mathbb{C}(m)$, the quantity $\left(1-s+\mu_{\tilde{\pi}}(j)\right) / 2$ is away from all poles of $\Gamma(s)$ by at least $(16 m)^{-1}$. For $j=1, \ldots, m$, denote by $\beta(j)$ the fractional part of $\Re e \mu_{\widetilde{\pi}}(j)$. In addition, let $\beta(0)=0$ and $\beta(m+1)=1$. Then all $\beta(j) \in[0,1]$, and hence there exist $\beta\left(j_{1}\right), \beta\left(j_{2}\right)$ such that $\beta\left(j_{2}\right)-\beta\left(j_{1}\right) \geqslant 1 /(3 m)$ and there is no $\beta(j)$ lying between $\beta\left(j_{1}\right)$ and $\beta\left(j_{2}\right)$. Consequently, for all $n=0,1,2, \ldots$, the strips

$$
S_{-n}:=\left\{s \in \mathbb{C}:-n+\beta\left(j_{1}\right)+(8 m)^{-1} \leqslant \Re e s \leqslant-n+\beta\left(j_{2}\right)-(8 m)^{-1}\right\}
$$

are subsets of $\mathbb{C}(m)$.

The following assertions (i) and (ii) are Lemma 4.3(d) and Lemma 4.4 of [7], respectively.

Lemma 2.1. Let $\pi$ be an irreducible unitary cuspidal representation of $G L_{m}\left(\mathbb{A}_{\mathbb{Q}}\right)$ with $m \geqslant 2$.

(i) For $T \geqslant 2$, there exists $\tau_{T}$ with $T \leqslant \tau_{T} \leqslant T+1$ such that

$$
-\frac{L^{\prime}}{L}\left(\sigma \pm \mathrm{i} \tau_{T}, \pi\right) \ll \log ^{2}\left(Q_{\pi} T\right) \quad(|\sigma| \leqslant 2) .
$$

(ii) If $s$ is in some strip $S_{-n}$ as in (2.3) with $n \geqslant 2$, then

$$
-\frac{L^{\prime}}{L}(s, \pi) \ll 1 .
$$

The implied constants depend only on $m$.

The next lemma is about the distribution of zeros of $L(s, \pi)$. For its proof, one is referred to Lemma 4.3 of Liu \& Ye [7], or Theorem 5.8 of Iwaniec \& Kowalski [3]. 
Lemma 2.2. Let $\pi$ be an irreducible unitary cuspidal representation of $G L_{m}\left(\mathbb{A}_{\mathbb{Q}}\right)$ with $m \geqslant 2$. All the nontrivial zeros of $\Phi(s, \pi)$ are in the critical strip $0 \leqslant \sigma \leqslant 1$. Let $N(T, \pi)$ be the number of its nontrivial zeros within the rectangular $0 \leqslant \sigma \leqslant 1$ and $|\tau| \leqslant T$. Then

$$
N(T, \pi) \ll T \log \left(Q_{\pi} T\right)
$$

and

$$
N(T+1, \pi)-N(T, \pi) \ll \log \left(Q_{\pi} T\right),
$$

where the implied constants are absolute.

\section{An explicit FORMula}

Explicit formulae of different forms were established by many authors. In particular, under GRC, explicit formulae for general $L$-functions were proved in [3, (5.53)]. The explicit formula below is unconditional, and plays a key role in the proofs of Theorems 1 and 2 .

Lemma 3.1. Let $\pi$ be an irreducible unitary cuspidal representation of $G L_{m}\left(\mathbb{A}_{\mathbb{Q}}\right)$ with $m \geqslant 2$, and $A>0$. Then, for $x \geqslant 2$ and $2 \leqslant T \leqslant x^{A}$, we have

$$
\psi(x, \pi)=-\sum_{|\gamma| \leqslant T} \frac{x^{\rho}}{\rho}-\sum_{\substack{\kappa^{\prime}<\lambda<\kappa \\|\nu| \leqslant T}} \frac{x^{\mu}}{\mu}-\frac{L^{\prime}}{L}(0, \pi)+O\left(R_{\pi}(x, T)\right),
$$

where

$$
R_{\pi}(x, T):=\sum_{|n-x| \leqslant x / \sqrt{T}}\left|\Lambda_{\pi}(n)\right|+\frac{x\left(\log Q_{\pi}\right) \log \left(Q_{\pi} x\right)}{\sqrt{T}}+\frac{x \log ^{2}\left(Q_{\pi} x\right)}{T}+\frac{\log T}{x},
$$

$-2<\kappa^{\prime}<-1, \kappa=1+1 / \log x$, and $\mu$ (resp. $\left.\rho\right)$ goes over the trivial zeros $\mu=\lambda+\mathrm{i} \nu$ (resp. the nontrivial zeros $\rho=\beta+\mathrm{i} \gamma$ ) of $L(s, \pi)$. The implied constant depends only on $A$ and $m$.

Proof. Since the series (1.13) converges absolutely for $\sigma>1$, we can apply the Perron formula [17, Theorem II.2.2] with $\kappa=1+1 / \log x$, so that

$$
\psi(x, \pi)=\frac{1}{2 \pi \mathrm{i}} \int_{\kappa-\mathrm{i} T}^{\kappa+\mathrm{i} T}-\frac{L^{\prime}}{L}(s, \pi) \frac{x^{s}}{s} \mathrm{~d} s+O\left(x^{\kappa} \sum_{n=1}^{\infty} \frac{\left|\Lambda_{\pi}(n)\right|}{n^{\kappa}(1+T|\log (x / n)|)}\right) .
$$

In order to treat the $O$-term, we split the sum into two parts according to

$$
|x-n| \leqslant x / \sqrt{T} \quad \text { or } \quad|x-n|>x / \sqrt{T} \text {. }
$$

By the Cauchy-Schwarz inequality, it follows that

$$
\begin{aligned}
\sum_{|x-n|>x / \sqrt{T}} \frac{\left|\Lambda_{\pi}(n)\right|}{n^{\kappa}(1+T|\log (x / n)|)} & \ll \frac{1}{\sqrt{T}} \sum_{n=1}^{\infty} \frac{\left|\Lambda_{\pi}(n)\right|}{n^{\kappa}} \\
& \ll \frac{1}{\sqrt{T}}\left(\sum_{n=1}^{\infty} \frac{\left|\Lambda_{\pi}(n)\right|^{2}}{n^{\kappa}}\right)^{1 / 2}\left(\sum_{n=1}^{\infty} \frac{1}{n^{\kappa}}\right)^{1 / 2} .
\end{aligned}
$$


According to $[3,(5.48)]$, we have

$$
\sum_{n \leqslant u}\left|\Lambda_{\pi}(n)\right|^{2} \ll m^{2} u \log ^{2}\left(Q_{\pi} u\right) \quad(u \geqslant 1),
$$

where the implied constant is absolute. Thus a simple integration by parts gives us

$$
\begin{aligned}
\sum_{n=1}^{\infty} \frac{\left|\Lambda_{\pi}(n)\right|^{2}}{n^{\kappa}} & =\int_{1-}^{\infty} \frac{1}{u^{\kappa}} \mathrm{d}\left(\sum_{n \leqslant u}\left|\Lambda_{\pi}(n)\right|^{2}\right) \\
& \ll_{m} \int_{1}^{\infty} \frac{\log ^{2}\left(Q_{\pi} u\right)}{u^{\kappa}} \mathrm{d} u \\
& \ll_{m} \frac{\log ^{2} Q_{\pi}}{\kappa-1}+\frac{1}{(\kappa-1)^{3}} .
\end{aligned}
$$

Similarly but more easily, we have

$$
\sum_{n=1}^{\infty} \frac{1}{n^{\kappa}} \ll \frac{1}{\kappa-1}
$$

Combining these estimates, we can find that

$$
x^{\kappa} \sum_{|x-n|>x / \sqrt{T}} \frac{\left|\Lambda_{\pi}(n)\right|}{n^{\kappa}(1+T|\log (x / n)|)} \ll \frac{x(\log x) \log \left(Q_{\pi} x\right)}{\sqrt{T}} .
$$

Next, we shall evaluate the integral on the right-hand side of (3.2). For this purpose, we shift the contour of integration to the left. Choose $\kappa^{\prime}$ with $-2<\kappa^{\prime}<-1$ such that the vertical line $\sigma=\kappa^{\prime}$ is contained in the strip $S_{-2} \subset \mathbb{C}(m)$; this is guaranteed by the structure of $\mathbb{C}(m)$. Without loss of generality, let $T \geqslant 2$ be a large number such that $T$ and $-T$ can be taken as the $\tau_{T}$ in Lemma 2.1(i). Now we consider the contour $\mathscr{L}_{1} \cup \mathscr{L}_{2} \cup \mathscr{L}_{3}$ with

$$
\mathscr{L}_{1}:=\left[\kappa^{\prime}-\mathrm{i} T, \kappa-\mathrm{i} T\right], \quad \mathscr{L}_{2}:=\left[\kappa^{\prime}-\mathrm{i} T, \kappa^{\prime}+\mathrm{i} T\right], \quad \mathscr{L}_{3}:=\left[\kappa^{\prime}+\mathrm{i} T, \kappa+\mathrm{i} T\right] .
$$

By Lemma 2.2 and (2.1), certain nontrivial zeros $\rho=\beta+\mathrm{i} \gamma$ and trivial zeros $\mu=\lambda+\mathrm{i} \nu$ of $L(s, \pi)$, as well as $s=0$ are passed by the shifting of the contour.

Computing the residues, we have

$$
\begin{aligned}
\frac{1}{2 \pi \mathrm{i}} \int_{\kappa-\mathrm{i} T}^{\kappa+\mathrm{i} T}-\frac{L^{\prime}}{L}(s, \pi) \frac{x^{s}}{s} \mathrm{~d} s= & -\sum_{|\gamma| \leqslant T} \frac{x^{\rho}}{\rho}-\sum_{\substack{\kappa^{\prime}<\lambda<\kappa \\
|\nu| \leqslant T}} \frac{x^{\mu}}{\mu}-\frac{L^{\prime}}{L}(0, \pi) \\
& -\frac{1}{2 \pi \mathrm{i}} \int_{\mathscr{L}_{1} \cup \mathscr{L}_{2} \cup \mathscr{L}_{3}}-\frac{L^{\prime}}{L}(s, \pi) \frac{x^{s}}{s} \mathrm{~d} s .
\end{aligned}
$$

The integral on $\mathscr{L}_{1}$ can be estimated by Lemma 2.1(i) as

$$
\begin{aligned}
\frac{1}{2 \pi \mathrm{i}} \int_{\mathscr{L}_{1}}-\frac{L^{\prime}}{L}(s, \pi) \frac{x^{s}}{s} \mathrm{~d} s & \ll \int_{\kappa^{\prime}}^{\kappa} \log ^{2}\left(Q_{\pi} T\right) \frac{x^{\sigma}}{T} \mathrm{~d} \sigma \\
& \ll \frac{x \log ^{2}\left(Q_{\pi} T\right)}{T},
\end{aligned}
$$


and the same upper bound also holds for the integral on $\mathscr{L}_{3}$. By Lemma 2.1(ii), then

$$
\begin{aligned}
\frac{1}{2 \pi \mathrm{i}} \int_{\mathscr{L}_{2}}-\frac{L^{\prime}}{L}(s, \pi) \frac{x^{s}}{s} \mathrm{~d} s & \ll \int_{-T}^{T} \frac{x^{\kappa^{\prime}}}{|\tau|+1} \mathrm{~d} t \\
& \ll \frac{\log T}{x} .
\end{aligned}
$$

Therefore, (3.5) becomes

$$
\begin{aligned}
\frac{1}{2 \pi \mathrm{i}} \int_{\kappa-\mathrm{i} T}^{\kappa+\mathrm{i} T}-\frac{L^{\prime}}{L}(s, \pi) \frac{x^{s}}{s} \mathrm{~d} s= & -\sum_{\substack{|\gamma| \leqslant T \\
\rho}} \frac{x^{\rho}}{\rho}-\sum_{\substack{\kappa^{\prime}<\lambda<\kappa \\
|\nu| \leqslant T}} \frac{x^{\mu}}{\mu}-\frac{L^{\prime}}{L}(0, \pi) \\
& +O\left(\frac{x(\log x) \log \left(Q_{\pi} x\right)}{\sqrt{T}}+\frac{x \log ^{2}\left(Q_{\pi} T\right)}{T}+\frac{\log T}{x}\right) .
\end{aligned}
$$

Inserting the above formula and (3.4) into (3.2), we obtain the required result.

\section{Gallagher lemma And Proof of Theorem 1}

Our main tool is the following lemma of Gallagher [1, Lemma 1].

Lemma 4.1. Let $U>0$ and $\delta=\vartheta / U$ with $0<\vartheta<1$, and let

$$
S(u):=\sum_{\nu} c(\nu) \mathrm{e}^{2 \pi \mathrm{i} \nu u}
$$

be absolutely convergent, where the coefficients $c(\nu) \in \mathbb{C}$, and the frequencies of $\nu$ run over an arbitrary sequence of real numbers. Then

$$
\int_{-U}^{U}|S(u)|^{2} \mathrm{~d} u \ll_{\vartheta} U^{2} \int_{-\infty}^{+\infty}\left|\sum_{t<\nu \leqslant t+\delta} c(\nu)\right|^{2} \mathrm{~d} t
$$

Now we prove Theorem 1 . Let $10^{4} \leqslant X \leqslant x \leqslant 2 X$, and take $T=(X \log X)^{2}$ in the explicit formula (3.1) of Lemma 3.1. Since the length of the interval $(x-$ $x /(X \log X), x+x /(X \log X)]$ is $2 x /(X \log X) \leqslant 1 / 2$, this interval contains at most one integer; we denote this possible integer by $n_{x}$. Thus our explicit formula becomes

$$
\begin{aligned}
\psi(x, \pi)= & -\sum_{|\gamma| \leqslant(X \log X)^{2}} \frac{x^{\rho}}{\rho}-\sum_{\substack{\kappa^{\prime}<\lambda<\kappa \\
|\nu| \leqslant(X \log X)^{2}}} \frac{x^{\mu}}{\mu}-\frac{L^{\prime}}{L}(0, \pi) \\
& +O\left(\left|\Lambda_{\pi}\left(n_{x}\right)\right|+\log \left(Q_{\pi} X\right)+\frac{\left(\log Q_{\pi}\right)^{2}}{X(\log X)^{2}}\right),
\end{aligned}
$$

where the implied constant depends only on $m$. From this we can write

$$
\psi(x+h, \pi)-\psi(x, \pi)=A+B+O\left(C+\log \left(Q_{\pi} X\right)+\frac{\left(\log Q_{\pi}\right)^{2}}{X(\log X)^{2}}\right),
$$


PRIME NUMBER THEOREM IN SHORT INTERVALS FOR AUTOMORPHIC $L$-FUNCTIONS 9

where $h \leqslant 2 X \leqslant 2 x$ and

$$
\begin{aligned}
& A:=-\sum_{|\gamma| \leqslant(X \log X)^{2}} \frac{(x+h)^{\rho}-x^{\rho}}{\rho}, \\
& B:=-\sum_{\substack{\kappa^{\prime}<\lambda<\kappa \\
|\nu| \leqslant(X \log X)^{2}}} \frac{(x+h)^{\mu}-x^{\mu}}{\mu}, \\
& C:=\left|\Lambda_{\pi}\left(n_{x+h}\right)\right|+\left|\Lambda_{\pi}\left(n_{x}\right)\right| .
\end{aligned}
$$

Clearly,

$$
\begin{aligned}
\int_{X}^{2 X}|\psi(x+h, \pi)-\psi(x, \pi)|^{2} \mathrm{~d} x \ll & \int_{X}^{2 X}\left(|A|^{2}+|B|^{2}+|C|^{2}\right) \mathrm{d} x \\
& +X \log ^{2}\left(Q_{\pi} X\right)+\frac{\left(\log Q_{\pi}\right)^{4}}{X(\log X)^{4}} .
\end{aligned}
$$

We start from $A$. In $A$, we split the sum over $|\gamma|$ at $T$, with $4 \leqslant T \leqslant(X \log X)^{2}$ a parameter that will be specified later, and define

$$
S_{1}(y):=\sum_{|\gamma| \leqslant T} y^{\mathrm{i} \gamma} \quad \text { and } \quad S_{2}(y):=\sum_{T<|\gamma| \leqslant(X \log X)^{2}} \frac{y^{\mathrm{i} \gamma}}{\rho} .
$$

Under GRH, the sum in $A$ runs over the nontrivial zeros $\rho=1 / 2+\mathrm{i} \gamma$ of $L(s, \pi)$ with $|\gamma|$ up to $(X \log X)^{2}$. Thus we can write

$$
\begin{aligned}
A & =-\sum_{|\gamma| \leqslant T} \frac{(x+h)^{\rho}-x^{\rho}}{\rho}-\sum_{T<|\gamma| \leqslant(X \log X)^{2}} \frac{(x+h)^{\rho}-x^{\rho}}{\rho} \\
& =-\sum_{|\gamma| \leqslant T} \int_{x}^{x+h} y^{\rho-1} \mathrm{~d} y-\sum_{T<|\gamma| \leqslant(X \log X)^{2}} \frac{(x+h)^{1 / 2+\mathrm{i} \gamma}-x^{1 / 2+\mathrm{i} \gamma}}{\rho} \\
& =-\int_{x}^{x+h} \frac{S_{1}(y)}{y^{1 / 2}} \mathrm{~d} y-(x+h)^{1 / 2} S_{2}(x+h)+x^{1 / 2} S_{2}(x) \\
& =: A_{1}+A_{2}+A_{3},
\end{aligned}
$$

say. By the Cauchy-Schwarz inequality,

$$
\left|A_{1}\right|^{2} \leqslant h \int_{x}^{x+h} \frac{\left|S_{1}(y)\right|^{2}}{y} \mathrm{~d} y
$$

In view of $h \leqslant 2 X$, the contribution from $\left|A_{1}\right|^{2}$ is estimated as

$$
\int_{X}^{2 X}\left|A_{1}\right|^{2} \mathrm{~d} x \ll h \int_{X}^{2 X}\left(\int_{x}^{x+h} \frac{\left|S_{1}(y)\right|^{2}}{y} \mathrm{~d} y\right) \mathrm{d} x \ll h^{2} \int_{X}^{4 X} \frac{\left|S_{1}(y)\right|^{2}}{y} \mathrm{~d} y .
$$


Changing variable $y=X \mathrm{e}^{2 \pi u}$ and applying Lemma 4.1 and (2.5) of Lemma 2.2, it follows that

$$
\begin{aligned}
\int_{X}^{2 X}\left|A_{1}\right|^{2} \mathrm{~d} x & \ll h^{2} \int_{0}^{(\log 2) / \pi}\left|\sum_{|\gamma| \leqslant T} X^{\mathrm{i} \gamma} \mathrm{e}^{2 \pi \mathrm{i} \gamma u}\right|^{2} \mathrm{~d} u \\
& \ll h^{2} \int_{-\infty}^{+\infty}\left(\sum_{|\gamma| \leqslant T, t<\gamma \leqslant t+1} 1\right)^{2} \mathrm{~d} t \\
& \ll h^{2} \int_{0}^{T}\left(\sum_{t<\gamma \leqslant t+1} 1\right)^{2} \mathrm{~d} t \\
& \ll h^{2} T \log ^{2}\left(Q_{\pi} T\right) .
\end{aligned}
$$

The contribution from $\left|A_{2}\right|^{2}$ can be estimated as

$$
\begin{aligned}
\int_{X}^{2 X}\left|A_{2}\right|^{2} \mathrm{~d} x & \ll X^{2} \int_{X}^{4 X} \frac{\left|S_{2}(x)\right|^{2}}{x} \mathrm{~d} x \\
& =X^{2} \int_{0}^{(\log 2) / \pi}\left|\sum_{T<|\gamma| \leqslant(X \log X)^{2}} \frac{X^{\mathrm{i} \gamma}}{\rho} \mathrm{e}^{2 \pi \mathrm{i} \gamma u}\right|^{2} \mathrm{~d} u \\
& \ll X^{2} \int_{-\infty}^{+\infty}\left(\sum_{T<|\gamma| \leqslant(X \log X)^{2}, t<\gamma<t+1} \frac{1}{|\gamma|}\right)^{2} \mathrm{~d} t \\
& \ll X^{2} \int_{T-1}^{(X \log X)^{2}}\left(\sum_{t<\gamma \leqslant t+1} \frac{1}{|\gamma|}\right)^{2} \mathrm{~d} t .
\end{aligned}
$$

By using (2.5) and (2.4) of Lemma 2.2, a simple integration by parts gives us

$$
\sum_{t<\gamma \leqslant t+1} \frac{1}{|\gamma|}=\int_{t}^{t+1} \frac{1}{u} \mathrm{~d} N(u, \pi) \ll \frac{\log \left(Q_{\pi} t\right)}{t} .
$$

Thus

$$
\int_{X}^{2 X}\left|A_{2}\right|^{2} \mathrm{~d} x \ll X^{2} \int_{T-1}^{(X \log X)^{2}} \frac{\log ^{2}\left(Q_{\pi} t\right)}{t^{2}} \mathrm{~d} t \ll \frac{X^{2} \log ^{2}\left(Q_{\pi} T\right)}{T} .
$$

Similarly, after taking $x+h=y$, we have

$$
\int_{X}^{2 X}\left|A_{3}\right|^{2} \mathrm{~d} x \ll \frac{X^{2} \log ^{2}\left(Q_{\pi} T\right)}{T} .
$$

We conclude from (4.2), (4.4) and (4.5) that

$$
\int_{X}^{2 X}|A|^{2} \mathrm{~d} x \ll h^{2} T \log ^{2}\left(Q_{\pi} T\right)+\frac{X^{2} \log ^{2}\left(Q_{\pi} T\right)}{T} .
$$


PRIME NUMBER THEOREM IN SHORT INTERVALS FOR AUTOMORPHIC $L$-FUNCTIONS11

For the mean-value of $|B|^{2}$, we apply (2.1) and (1.11), to get

$$
\begin{aligned}
\int_{X}^{2 X}|B|^{2} \mathrm{~d} x & =\int_{X}^{2 X}\left|\sum_{\substack{\kappa^{\prime}<\lambda<\kappa \\
|\nu| \leqslant(X \log X)^{2}}} \frac{(x+h)^{\mu}-x^{\mu}}{\mu}\right|^{2} \mathrm{~d} x \\
& \ll \int_{X}^{2 X}\left(\sum_{\substack{\kappa^{\prime}<\lambda<\kappa \\
|\nu| \leqslant(X \log X)^{2}}} x^{\lambda-1} h\right)^{2} \mathrm{~d} x \\
& \ll \int_{X}^{2 X}\left(x^{\theta-1} h\right)^{2} \mathrm{~d} x \\
& \ll h^{2} .
\end{aligned}
$$

It remains to estimate the contribution of $|C|^{2}$. We have

$$
\begin{aligned}
\int_{X}^{2 X}|C|^{2} \mathrm{~d} x & =\int_{X}^{2 X}\left(\left|\Lambda_{\pi}\left(n_{x+h}\right)\right|+\left|\Lambda_{\pi}\left(n_{x}\right)\right|\right)^{2} \mathrm{~d} x \\
& \ll \sum_{j=[X]}^{[2 X]} \int_{j}^{j+1}\left(\left|\Lambda_{\pi}\left(n_{x+h}\right)\right|^{2}+\left|\Lambda_{\pi}\left(n_{x}\right)\right|^{2}\right) \mathrm{d} x .
\end{aligned}
$$

Since $h(x)$ is increasing and $h(x) \leqslant x$, we have trivially, for $j \leqslant x \leqslant j+1$, that

$$
j-1 \leqslant n_{x+h(x)} \leqslant 2(j+2), \quad j-1 \leqslant n_{x} \leqslant j+2 .
$$

Thus,

$$
\int_{X}^{2 X}|C|^{2} \mathrm{~d} x \ll \sum_{j=[X]-1}^{3[2 X]}\left|\Lambda_{\pi}(j)\right|^{2} \ll X \log ^{2}\left(Q_{\pi} X\right),
$$

by applying (3.3).

Finally inserting (4.6), (4.7) and (4.8) to (4.1), and taking $T=X / h(2 X)$, we find

$$
\int_{X}^{2 X}|\psi(x+h, \pi)-\psi(x, \pi)|^{2} \mathrm{~d} x \ll h(2 X) X \log ^{2}\left(Q_{\pi} X\right)+\frac{\left(\log Q_{\pi}\right)^{4}}{X(\log X)^{4}}
$$

for any increasing function $h(x)$ satisfying $1 \leqslant h(x) \leqslant x$. A splitting-up argument then gives the required inequality (1.17). This completes the proof of Theorem 1.

\section{PAir Correlation of ZERos AND PROOF of Theorem 2}

The proof of Theorem 2 is very similar to that of Theorem 1 . The only difference is to estimate the contribution of $\left|A_{i}\right|^{2}$ with the help of hypothesis (1.19) instead of Gallagher's lemma and Lemma 2.2. We retain the notation in Section 4. According to the first line of (4.2), we have

$$
\int_{X}^{2 X}\left|A_{1}\right|^{2} \mathrm{~d} x \ll h^{2} \int_{0}^{(\log 2) / \pi}\left|\sum_{|\gamma| \leqslant T} X^{\mathrm{i} \gamma} \mathrm{e}^{2 \pi \mathrm{i} \gamma u}\right|^{2} \mathrm{~d} u .
$$


In view of the trivial inequality $\mathrm{e}^{-4 \pi|u|} \gg 1(0 \leqslant u \leqslant 1)$ and the classical formula

$$
2 \pi \int_{-\infty}^{+\infty} \mathrm{e}^{-4 \pi|u|+2 \pi \mathrm{i} t u} \mathrm{~d} u=W(t)
$$

we can deduce

$$
\begin{aligned}
\int_{X}^{2 X}\left|A_{1}\right|^{2} \mathrm{~d} x & \ll h^{2} \int_{0}^{(\log 2) / \pi} \mathrm{e}^{-4 \pi|u|}\left|\sum_{|\gamma| \leqslant T} X^{\mathrm{i} \gamma} \mathrm{e}^{2 \pi \mathrm{i} \gamma u}\right|^{2} \mathrm{~d} u \\
& \ll h^{2} \int_{-\infty}^{+\infty} \mathrm{e}^{-4 \pi|u|}\left|\sum_{0<\gamma \leqslant T} X^{\mathrm{i} \gamma} \mathrm{e}^{2 \pi \mathrm{i} \gamma u}\right|^{2} \mathrm{~d} u \\
& \ll h^{2} \sum_{0<\gamma_{1}, \gamma_{2} \leqslant T} X^{\mathrm{i}\left(\gamma_{1}-\gamma_{2}\right)} \int_{-\infty}^{+\infty} \mathrm{e}^{-4 \pi|u|} \mathrm{e}^{2 \pi \mathrm{i}\left(\gamma_{1}-\gamma_{2}\right) u} \mathrm{~d} u \\
& \ll h^{2} \sum_{0<\gamma_{1}, \gamma_{2} \leqslant T} X^{\mathrm{i}\left(\gamma_{1}-\gamma_{2}\right)} W\left(\gamma_{1}-\gamma_{2}\right) \\
& =h^{2} F_{T}^{\pi}\left(\frac{\log X}{2 \pi}\right) .
\end{aligned}
$$

Assuming (1.19), we have

$$
\int_{X}^{2 X}\left|A_{1}\right|^{2} \mathrm{~d} x \ll h^{2} T \log \left(Q_{\pi} T\right) .
$$

Next we estimate the contribution of $\left|A_{2}\right|^{2}$. By partial summation, it follows that

$$
\begin{aligned}
\sum_{T<\gamma \leqslant(X \log X)^{2}} \frac{X^{\mathrm{i} \gamma}}{\rho} \mathrm{e}^{2 \pi \mathrm{i} \gamma u} & =\int_{T}^{(X \log X)^{2}} \frac{1}{1 / 2+\mathrm{i} t} \mathrm{~d}\left(\sum_{\gamma \leqslant t} X^{\mathrm{i} \gamma} \mathrm{e}^{2 \pi \mathrm{i} \gamma u}\right) \\
= & \sum_{\gamma \leqslant(X \log X)^{2}} \frac{X^{\mathrm{i} \gamma} \mathrm{e}^{2 \pi \mathrm{i} \gamma u}}{1 / 2+\mathrm{i}(X \log X)^{2}}-\sum_{\gamma \leqslant T} \frac{X^{\mathrm{i} \gamma} \mathrm{e}^{2 \pi \mathrm{i} \gamma u}}{1 / 2+\mathrm{i} T} \\
& +\mathrm{i} \int_{T}^{(X \log X)^{2}} \sum_{\gamma \leqslant t} X^{\mathrm{i} \gamma} \mathrm{e}^{2 \pi \mathrm{i} \gamma u} \frac{\mathrm{d} t}{(1 / 2+\mathrm{i} t)^{2}} .
\end{aligned}
$$

By the Cauchy-Schwarz inequality, we have

$$
\begin{aligned}
& \left(\int_{T}^{(X \log X)^{2}}\left|\sum_{\gamma \leqslant t} X^{\mathrm{i} \gamma} \mathrm{e}^{2 \pi \mathrm{i} \gamma u}\right| \frac{\mathrm{d} t}{t^{2}}\right)^{2} \\
& \ll \int_{T}^{(X \log X)^{2}} \frac{\mathrm{d} t}{t(\log (1+t / T))^{2}} \int_{T}^{(X \log X)^{2}}\left|\sum_{\gamma \leqslant t} X^{\mathrm{i} \gamma} \mathrm{e}^{2 \pi \mathrm{i} \gamma u}\right|^{2} \frac{(\log (1+t / T))^{2}}{t^{3}} \mathrm{~d} t \\
& \ll \int_{T}^{(X \log X)^{2}}\left|\sum_{\gamma \leqslant t} X^{\mathrm{i} \gamma} \mathrm{e}^{2 \pi \mathrm{i} \gamma u}\right|^{2} \frac{(\log (1+t / T))^{2}}{t^{3}} \mathrm{~d} t .
\end{aligned}
$$


PRIME NUMBER THEOREM IN SHORT INTERVALS FOR AUTOMORPHIC $L$-FUNCTIONS13

Thus

$$
\begin{aligned}
\left|\sum_{T<\gamma \leqslant(X \log X)^{2}} \frac{X^{\mathrm{i} \gamma}}{\rho} \mathrm{e}^{2 \pi \mathrm{i} \gamma u}\right|^{2} \ll & \frac{1}{X^{4}}\left|\sum_{\gamma \leqslant(X \log X)^{2}} X^{\mathrm{i} \gamma} \mathrm{e}^{2 \pi \mathrm{i} \gamma u}\right|^{2}+\frac{1}{T^{2}}\left|\sum_{\gamma \leqslant T} X^{\mathrm{i} \gamma} \mathrm{e}^{2 \pi \mathrm{i} \gamma u}\right|^{2} \\
& +\int_{T}^{(X \log X)^{2}}\left|\sum_{\gamma \leqslant t} X^{\mathrm{i} \gamma} \mathrm{e}^{2 \pi \mathrm{i} \gamma u}\right|^{2} \frac{(\log (1+t / T))^{2}}{t^{3}} \mathrm{~d} t .
\end{aligned}
$$

In view of the first two lines of (4.3) and the estimate above, we can write

$$
\begin{aligned}
\int_{X}^{2 X}\left|A_{2}\right|^{2} \mathrm{~d} x \ll & X^{2}\left\{\frac{1}{X^{4}} \int_{0}^{(\log 2) / \pi}\left|\sum_{\gamma \leqslant(X \log X)^{2}} X^{\mathrm{i} \gamma} \mathrm{e}^{2 \pi \mathrm{i} \gamma u}\right|^{2} \mathrm{~d} u\right. \\
& +\frac{1}{T^{2}} \int_{0}^{(\log 2) / \pi}\left|\sum_{\gamma \leqslant T} X^{\mathrm{i} \gamma} \mathrm{e}^{2 \pi \mathrm{i} \gamma u}\right|^{2} \mathrm{~d} u \\
& \left.+\int_{T}^{(X \log X)^{2}}\left(\int_{0}^{(\log 2) / \pi}\left|\sum_{\gamma \leqslant t} X^{\mathrm{i} \gamma} \mathrm{e}^{2 \pi \mathrm{i} \gamma u}\right|^{2} \mathrm{~d} u\right) \frac{(\log (1+t / T))^{2}}{t^{3}} \mathrm{~d} t\right\} .
\end{aligned}
$$

From this, a similar argument to (5.1) allows us to deduce

$$
\begin{aligned}
& \int_{X}^{2 X}\left|A_{2}\right|^{2} \mathrm{~d} x \\
& \ll X^{2}\left(\frac{1}{T}+\int_{T}^{(X \log X)^{2}} \frac{(\log (1+t / T))^{2}}{t^{2}} \mathrm{~d} t\right) \sup _{T \leqslant t \leqslant(X \log X)^{2}} \frac{1}{t} F_{t}^{\text {Auto }}\left(\frac{\log X}{2 \pi}\right) \\
& \ll \frac{X^{2}}{T} \sup _{T \leqslant t \leqslant(X \log X)^{2}} \frac{1}{t} F_{t}^{\text {Auto }}\left(\frac{\log X}{2 \pi}\right) .
\end{aligned}
$$

Assuming (1.19), it follows that

$$
\int_{X}^{2 X}\left|A_{2}\right|^{2} \mathrm{~d} x \ll \frac{X^{2}}{T} \sup _{T \leqslant t \leqslant(X \log X)^{2}} \log \left(Q_{\pi} t\right) \ll \frac{X^{2} \log \left(Q_{\pi} X\right)}{T} .
$$

The same estimate also holds for $\int_{X}^{2 X}\left|A_{3}\right|^{2} \mathrm{~d} x$.

From these conclusions and (5.2), we get that

$$
\int_{X}^{2 X}|A|^{2} \mathrm{~d} x \ll h^{2} T \log \left(Q_{\pi} T\right)+\frac{X^{2} \log \left(Q_{\pi} X\right)}{T} .
$$

Finally, inserting (5.3), (4.7), and (4.8) to (4.1), and taking $T=X h(2 X)^{-1}$, we find that

$$
\int_{X}^{2 X}|\psi(x+h, \pi)-\psi(x, \pi)|^{2} \mathrm{~d} x \ll h(2 X) X \log \left(Q_{\pi} X\right)+X \log ^{2}\left(Q_{\pi} X\right)+\frac{\left(\log Q_{\pi}\right)^{4}}{X(\log X)^{4}}
$$

for any increasing functions $h(x)$ satisfying $1 \leqslant h(x) \leqslant x$. A splitting-up argument then gives the required inequality (1.20). This completes the proof of Theorem 2. 


\section{REFERENCES}

[1] P. X. Gallagher, A large sieve density estimate near $\sigma=1$, Invent. Math. 11 (1970), 329-339.

[2] D. R. Heat-Brown, Gaps between primes, and the pair correction of zeros of the zeta-function, Acta Arith. 16 (1082), 85-99.

[3] H. Iwaniec \& E. Kowalski, Analytic Number Theory, Amer. Math. Soc. Colloquium Publ. 53, Amer. Math. Soc., Providence, 2004.

[4] H. Iwaniec \& P. Sarnak, Perspectives in the analytic theory of L-functions, Geom. Funct. Anal. special issue (2000), 705-741.

[5] H. Jacquet \& J. A. Shalika, On Euler products and the classification of automorphic representations I, Amer. J. Math. 103 (1981), 499-558.

[6] Jianya Liu, Yan Qu \& Jie Wu, Two Linnik-type problems for automorphic L-functions, Math. Proc. Camb. Phil. Soc. 151 (2011), No. 2, 219-227.

[7] Jianya Liu \& Yangbo Ye, Superpositions of zeros of distinct L-functions, Forum Math. 14 (2002), 419-455.

[8] W. Luo, Z. Rudnick \& P. Sarnak, On Selberg's eigenvalue conjecture, Geom. Funct. Anal. 5 (1995), 387-401.

[9] H. L. Montgomery, The pair correlation of zeros of the zeta function, in: Analytic number theory (Proc. Sympos. Pure Math., Vol. XXIV, St. Louis Univ., St. Louis, Mo., 1972), pp. 181-193, Amer. Math. Soc., Providence, R.I., 1973.

[10] Yan Qu, The prime number theorem for automorphic L-functions for $G L_{m}$, J. Number Theory 122 (2007), 84-99.

[11] Yan Qu, Selberg's normal density theorem for automorphic L-functions for $G L_{m}$, Acta Math. Sin. (English Series) 23 (2007), 1903-1908.

[12] A. Selberg, On the normal sensity of primes in short intervals and the difference between consecutive primes, Archiv f. Math. og Naturvid. B 47 (1943), No. 6.

[13] F. Shahidi, On certain L-functions, Amer. J. Math. 103 (1981), 297-355.

[14] F. Shahidi, Fourier transforms of intertwining operators and Plancherel measures for $G L(n)$, Amer. J. Math. 106 (1984), 67-111.

[15] F. Shahidi, Local coefficients as Artin factors for real groups, Duke Math. J. 52 (1985), 973-1007.

[16] F. Shahidi, A proof of Langlands' conjecture on Plancherel measures; Complementary series for p-adic groups, Ann. Math. 132 (1990), 273-330.

[17] G. Tenenbaum, Introduction to analytic and probilistic number theory, Cambridge University Press, 1995.

Institute of Mathematics, Chinese Academy of Sciences, Beijing 100190, China

E-mail address: qukaren@gmail.com

School of Mathematics, Shandong University, Jinan, Shandong 250100, China, Institut Elie Cartan Nancy, CNRS, Université Henri Poincaré (Nancy 1), INRIA, 54506 VANDEUVRE-LÈs-NANCY, FRANCE

E-mail address: wujie@iecn.u-nancy.fr 